\title{
Review of The Psychobiology of Reproductive Behavior: An Evolutionary Perspective
}

\author{
MICHAEL J. MEANEY \\ McGill University, Douglas Hospital Research Centre, Verdun, Quebec, Canada
}

\section{The Psychobiology of Reproductive Behavior: An Evolutionary Perspective Edited by David Crews. 1987; Prentice-Hall. 350 pp. Paper, \$21.00.}

In 1959, Phoenix, Goy, Gerall, and Young published a landmark paper, in which they outlined a major hypothesis for the hormonal regulation of sexual behavior. In it, they described two ways in which hormones might regulate sexual expression. The first included organizational effects, in which perinatal exposure to selected hormones during specific periods in development organizes the neural circuitry of the brain and in so doing alters the nature or the magnitude of the response to certain stimuli (either hormonal or environmental) in later life. The second comprised activational effects, in which circulating hormones in adulthood alter the response of the animal to selected stimuli. This hypothesis has proved to be one of the most heuristic and fruitful ideas in the history of psychobiology. Since then, thousands of published papers from researchers throughout the world have been directed to the topic of the hormonal control of sexual behavior. Yet distressingly, the vast majority of these papers have focused on a very limited number of species and on an even more limited range of experimental paradigms, even though Goy, Beach, Phoenix, and other major figures in the earlier period of behavioral endocrinology used a variety of species and actually emphasized the importance of species variation.

The classical paradigm that has emerged from such studies involves the careful quantification of sexual behavior(s) of individual male and female laboratory rodents paired in small cages. There are several advantages to such a procedure, most notably the precision and the ease with which data can be collected. This has resulted in the accumulation of an enormous amount of data on the neuroendocrine regulation of sexual behavior and on the development of sexual behavior in rodents. It is likely that

Michael J. Meaney's mailing address is Department of Psychiatry, McGill University, Douglas Hospital Research Centre, 6875 La Salle Blvd, Verdun, Quebec H4H 1R3, Canada. most scientists are aware that the use of such a "sterile" testing environment facilitates behavioral analysis at the cost of sacrificing "ecological validity." Yet probably few are knowledgeable about the exact costs of this tradeoff. Perhaps even more worrisome is the fear that the training of younger scientists may occur in the absence of exposure to the merits of field research or at least of attempts to study behavior in seminaturalistic conditions. There continues to ensue a proliferation of research in behavioral endocrinology that represents rather minor variations on the theme of "sexologica rodentia" (see Svare, 1988). This point is rather reminiscent of the eloquent warning issued by Beach in 1950, with regard to the exaggerated focus directed towards the laboratory rat by behavioral scientists.

There are, however, a number of exceptions. Some researchers have explored varied species, and some have attempted to examine behavior in naturalistic environments or in seminaturalistic ones that resemble the conditions under which species-specific mating behaviors evolved. The Psychobiology of Reproductive Behavior presents a collection of chapters by a group of such researchers. Its chapters deal with many classes of animals, including fishes (Demski, Stacey), lizards (Crews, Moore), birds (Wingfield \& Moore, Silver \& Norgren), rodents (Bronson, McClintock), carnivores (Asa, Baum), ungulates (Asa), nonhuman primates (Sapolsky), and humans (Money), in experimental paradigms ranging from field studies to controlled laboratory experimentation. This mixture yields a fascinating picture of the regulation of mating and reproduction by a broad range of ecological and social stimuli transduced through diverse neuroendocrine mechanisms. The field of behavioral endocrinology would benefit greatly from more research like that described in this text.

A number of major themes emerge from this book: (1) the occurrence of species variation in the dependence of sexual behavior on the presence of circulating sex steroids and hypothalamic-pituitary releasing factors; (2) species variation (and within-species variation) in the degree to which the expression of sexual behavior is regulated by social stimuli; (3) the synchrony between the mat- 
ing behavior of the male and that of the female and the degree to which the behavior of the male is regulated by the female; (4) species variation in the degree of sexual dimorphism of behavior; and (5) the adaptations in mating patterns that have occurred across species in response to various environmental conditions. The editor clearly intended that this book should provide an example of an anthology in which common themes really do integrate the various chapters.

Crews makes one major point in the preface that is then underscored in each chapter that follows. He laments the "tendency for behavioral physiology and evolutionary biology to be mutually exclusive, almost opposite, endeavours." Thus, a number of chapters in the text clearly demonstrate that an evolutionary perspective can provide a useful approach for psychobiologists who seek to identify the physiological mechanisms that underlie reproductive behavior. To a certain extent, such neuroendocrine mechanisms can be logically inferred through a knowledge of the environment in which the behavior evolved. Thus, for example, the involvement of the superchiasmatic nuclei, a neuroanatomical transducer of information concerning photoperiodicity, in the regulation of seasonal breeders' mating behaviors is understandable. It is difficult to overstate the importance of this message in a field like neuroscience, where the bandwagon is clearly moving in the direction of reductionism. The point here is not merely that one needs to assess causality at various levels, but also that in so doing one might better understand the diverse molecular mechanisms that can influence the expression of behavior.

In general, the chapters are very clearly written. Each author has attempted to present the issues, both methodological and theoretical, that emerge from the research topic at hand. The result is a text that goes well beyond that of the conventional collection of individually authored chapters. The reader is presented with several excellent examples of how mating behaviors have evolved in relation to ecological and social demands and of how these environmental events are transduced by various neuroendocrine systems. Perhaps even more importantly, the reader gains a full appreciation of the variability both within species and across species in the nature of the regulation of mating behaviors. Considerable attention is given not only to the synchrony between sexual behavior and animals' environments, but also to the nature of the malefemale interactions involved in reproduction. The reader also gains a rich appreciation of how the sexual behavior of the male is often regulated and paced by the female (e.g., Wingfield \& Moore; McClintock). This influence has not always been fully apparent in data derived from more traditional experimental paradigms.

Perhaps one of the best features of this book is that it also enables the reader to appreciate the information that can be obtained through the complementary use of more controlled laboratory experimentation (see, e.g., Bronson; Baum; F. L. Moore). The reader is presented with a clear balance between the merits of field and laboratory approaches.

The text is well illustrated. The level of the material is appropriate for the desired readership (upper level undergraduates and new graduate students). The Psychobiology of Reproductive Behavior is an ideal, affordable companion text for courses in reproductive biology, and when the lecturer can assume a very basic knowledge of neuroendocrinology, the text will be suitable by itself. The book is also ideal for graduate seminars. Crews has certainly met his objectives in designing a readable, comprehensive, and stimulating work. I would strongly recommend it to any researcher in the field, including graduate students. It is also essential for all libraries.

\section{REFERENCES}

BEACH, F. A. (1950). The Snark was a Boojum. American Psychologist, 5, 115-124.

Phoenix, C. H., Goy, R. W., Gerall, A. A., \& Young, C. W. (1959). Organizing action of prenatally administered testosterone propionate on the tissues mediating mating behavior in the female guinea pig. Endocrinology, 65, 369-382.

SVARE, B. (1988). Some trends in the responses studied and the species employed by behavioral endocrinologists. Hormones \& Behavior, 22 , 139-142. 